\title{
Normas de comportamiento en salud y guías clínicas: del escrito a la aplicación
}

\section{Standards of behavior in health and clinical guidelines: from writing to the application}

Eduardo Lema F.1

\begin{abstract}
The adverse event in health continues to be an important cause of morbidity. Since the emergence of the safety culture, institutions, associations and collegiate bodies have been dedicated to the construction of standards, guidelines and protocols to improve patient safety, including adopting procedures and habits in an eclectic manner, from other professions and occupations. However, the human factor continues to be a frequent cause of adverse events, so it is worth reviewing some aspects of human behavior that help implement measures that reduce the gap between follow-up records of completed guidelines and actions actually carried out as a result of the adherence and implementation of clinical practice guidelines.
\end{abstract}

\section{RESUMEN}

El evento adverso en salud sigue siendo una causa importante de morbilidad. Desde la aparición de la cultura de seguridad, las instituciones, agremiaciones y cuerpos colegiados se han dedicado a la construcción de normas, guías y protocolos para mejorar la seguridad de los pacientes, incluso, adoptando procedimientos y hábitos de manera ecléctica, provenientes de otras profesiones y ocupaciones. Sin embargo, el factor humano sigue siendo una causa frecuente de eventos adversos, por lo cual vale la pena revisar algunos aspectos del comportamiento humano que ayuden a implementar medidas que disminuyan la brecha entre registros de seguimiento de guías diligenciadas y acciones efectivamente ejecutadas como consecuencia de la adherencia e implementación de guías de práctica clínica.

\section{Key words:}

Adverse event, adherence,

Safety in health care

\section{Palabras clave:}

Evento adverso, adherencia,

Seguridad en atención en salud

Médico Anestesiólogo. Profesor Asistente, Facultad de Salud, Departamento de Anestesiología, Universidad del Valle. Piloto Privado de Avión, Licencia 2765. Instructor de Escuela de Tierra. Licencia 1788.

Fecha de recepción: 10 de enero de 2019

Fecha de aceptación: 12 de enero de 2019

ORCID

https://orcid.org/0000-0002-3379-9774

Correspondencia:

Eduardo Lema Flórez

Email: eduardo.lema@correounivalle.edu.co 


\section{Introducción}

I 28 de noviembre de 2016, a las 21:59 horas, el avión de la empresa LaMia que cubría la ruta entre Santa Cruz (Bolivia) y el aeropuerto de Río Negro (Colombia) y que transportaba en su mayoría a los jugadores del equipo de fútbol Chapecoense de Brasil, se precipitó a tierra minutos antes del aterrizaje dejando 17 personas muertas y 6 heridas. Según la investigación realizada por las autoridades aeronáuticas de Colombia, Brasil, Bolivia, Estados Unidos e Inglaterra, la causa principal fue la falta de combustible suficiente para cubrir la ruta. Esta falta de combustible se debió no solo a una mala planificación del vuelo al no cumplir deliberadamente con la cantidad exigida para la ruta, sino también a la decisión del piloto en pleno vuelo de no reabastecer combustible en medio del trayecto por razones puramente financieras[1].

Desde la introducción del transporte en jet (motores de reacción) en los años cincuenta, los accidentes aéreos por causas mecánicas disminuyeron de manera considerable, sin embargo, los accidentes debidos a fallos humanos continúan ocurriendo. Las principales causas son deficiencias en la comunicación, en el trabajo en equipo y en la toma de decisiones[2] Figura 1. A partir de esto, en la aviación, se ha implementado la cultura de aceptar, cuestionar, reconocer las limitaciones del ser humano para de esta manera poder gestionar el riesgo. El segundo esfuerzo más importante fue la implementación de registros que recolecten la información sobre el cumplimiento o no de las normas a cargo tanto del piloto como de todo su equipo; el primero lo representan los procesos de selección. Un piloto no es seleccionado solamente por su deseo de volar una aeronave. Los procesos de selección escogen a los candidatos teniendo en cuenta sus capacidades técnicas y físicas y sobre todo, por su capacidad de comunicarse e integrar equipos capaces de lograr el objetivo, pues la falta de comunicación, de trabajo en equipo y los errores en la comunicación son factores que contribuyen en la génesis del evento adverso en la aviación[3]. Esta causalidad ha sido entendida igualmente en salud[4].

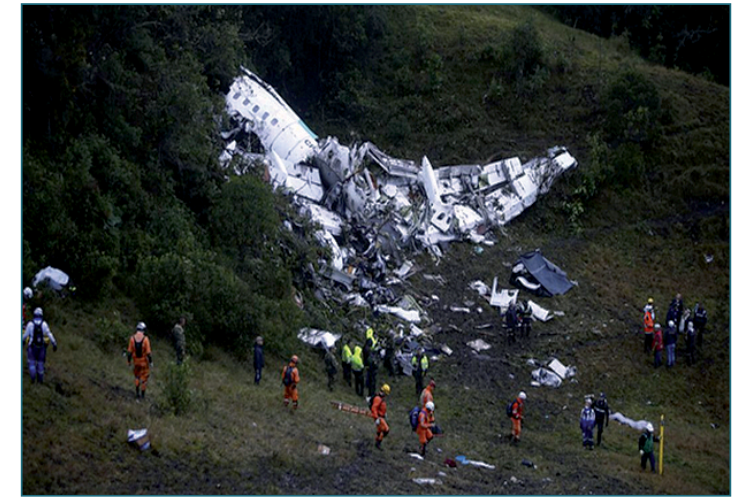

En salud, se han realizado esfuerzos para lograr implementar las actividades y medidas que han mejorado la seguridad en la aviación[5]: estrategias de apoyo como listas de chequeo, los manuales de decisión, las guías de práctica clínica y una fundamental Figura 2, el reporte del evento adverso. Sin embargo, los resultados, en términos de adherencia y de evento adverso, no parecen tener igual impacto, lo cual sugiere que dichas medidas, por si solas, podrían no ser extrapolables a la práctica sanitaria. Se requiere un mejor estudio del comportamiento humano, pues no solo con la implementación de una guía se obtendrá un efecto si el personal de salud no cree que esta contenga las mejores prácticas posibles o que su impacto en el paciente sea el esperado.

Una forma de medir el apego del personal de salud a las instrucciones institucionales puede representarlo una actividad básica que, además, es transversal a toda la línea de atención de un paciente: La historia clínica. Una historia clínica incompleta o mal diligenciada es un indicio de falta de adherencia al cumplimiento de normas. Elhalawani, en su trabajo publicado, encuentra que revisando los registros de anestesia, tanto en procedimientos como de urgencias, ninguno de los tópicos revisados (identificación, peso, edad, riesgo, vía aérea) tenía un diligenciamiento del cien por ciento y que el cumplimiento del registro fue menor en los procedimientos de emergencia

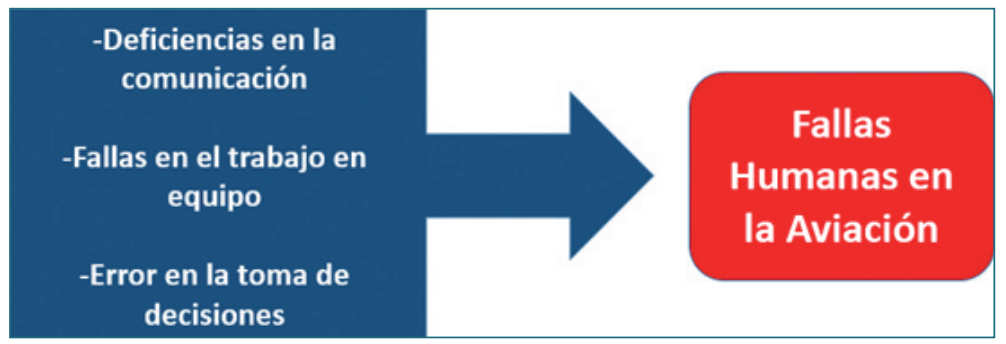

Figura 1. Causas principales de fallas humanas en la aviación. Construcción del autor. 


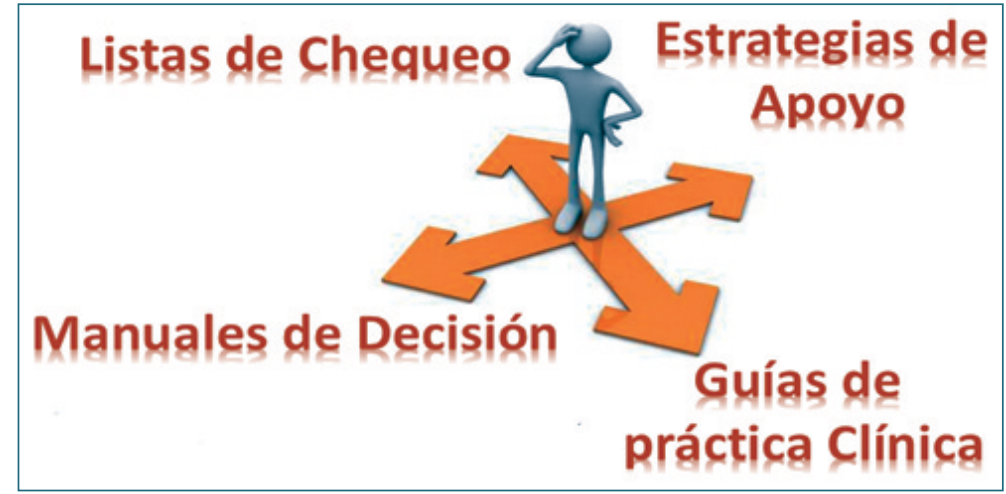

Figura 2. Estrategias de seguridad en salud tomadas de la aviación. Construcción del autor. que en los electivos. Igualmente, documenta Elhalawani cómo en los registros electrónicos se lograba mayor cumplimiento en las variables relacionadas con la identificación y con el procedimiento pero, en el registro manual, mayor cumplimiento de información clínica[6]. El impacto de una historia mal diligenciada no solo es de interés estadístico y/o legal. Un antecedente no registrado de reacción medicamentosa o de dificultad con algún procedimiento durante la atención puede favorecer la ocurrencia de un evento adverso posterior totalmente prevenible.

Desde los primeros trabajos de revisión en la búsqueda de las causas por las cuales los profesionales de la salud no tienen una adherencia alta a las guías de práctica clínica se han planteado cientos de razones, las cuales Lugtenberg agrupó en varias categorías[7]: Aquellas relacionadas con el conocimiento[8](recordación y/o familiaridad), aquellas relacionadas con el Comportamiento y con la actitud (inercia a la práctica previa, considerar que la aplicación no tendrá beneficios, falta de motivación, entre otras) (Figura 3).
A pesar de los ingentes esfuerzos de todas las instituciones de salud, de la amplia divulgación de las guías de práctica clínica, de volverlas incluso políticas institucionales y de calidad, la seguridad del paciente en términos de daños ocasionados con motivo de la atención sanitaria, en el 2018, sigue siendo reconocido como un problema de salud pública[9].

Se retoma entonces la pregunta: ¿Es suficiente con importar actividades realizadas en otras disciplinas para aumentar la seguridad de los pacientes? La probabilidad de que un pasajero sufra un accidente es de una en un millón de pasajeros, sin embargo, uno de cada 300 pacientes sufre algún daño por una atención en salud[9]. Además, de la diferencia en el proceso de selección de un piloto y en general del personal que tripulará una aeronave, existe una gran diferencia entre las dos disciplinas. Mientras un tripulante sufrirá las consecuencias de una mala decisión de seguridad al estar montado en el problema, el personal de salud jamás sufrirá físicamente las consecuencias de una mala decisión tomada en torno a la conducta o

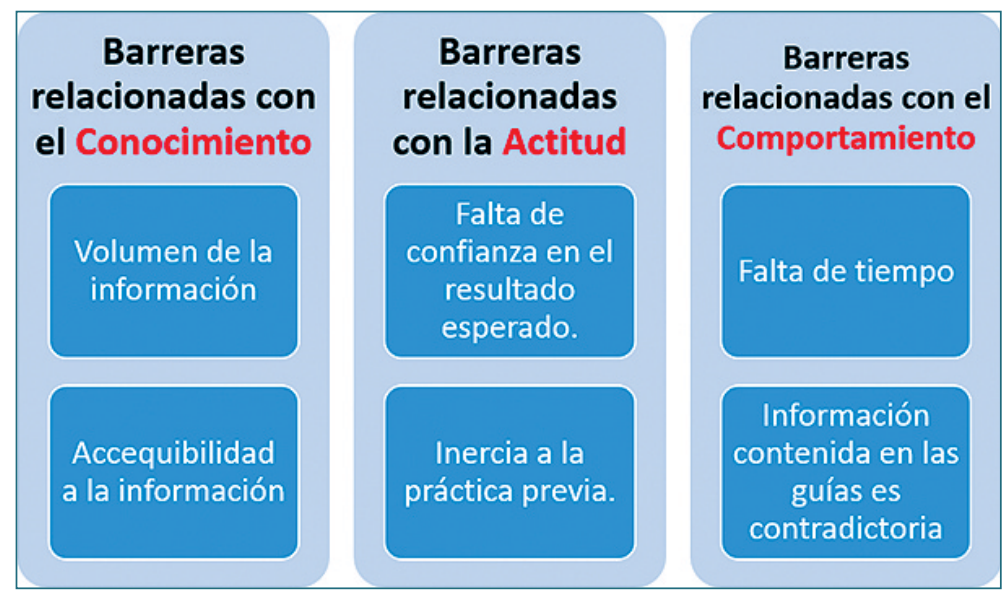

Figura 3. Barreras para la adherencia del personal de salud a las guías de práctica clínica[14]. Resumen construido por el autor. 
atención de una paciente. Esto es el reflejo de autocuidado. Una persona tiene naturalmente la tendencia a cuidar de sí mismo y, por ello, en condiciones normales, evitará exponerse a situaciones en las que su vida entre en peligro de manera voluntaria.

\section{¿Cuáles estrategias pueden ayudar a mejorar la seguridad?}

El primer paso es el proceso de selección. Un referente social del cumplimiento de instrucciones tanto de manera individual como colectiva, lo representa la vida militar. El proceso de selección en esta disciplina es cada vez menos arbitrario; hace más de 70 años abandonaron la entrevista personal tomando mayor importancia la valoración psicológica en los procesos de selección de las personas que entrarán a la vida militar[10]. En el ser humano existe una relación entre el cumplimiento de normas y la honestidad[11], y la sociedad definitivamente ejerce una gran influencia. Un contexto social en donde se violan los reglamentos afectará la honestidad de las personas en sus actividades diarias de tal manera que un individuo puede tener un comportamiento honesto o deshonesto, dependiendo no solo de sus condiciones y valores personales, sino también de su entorno, resultando más frecuente que muchas personas violen un poco los reglamentos a que unas pocas personas violen flagrantemente los mismos. Es decir, que en un proceso de selección se debe tratar de identificar aquella persona que jamás cumplirá las reglas, aquel que siempre las seguirá y lo más frecuente, la persona que lo hará, dependiendo de las circunstancias y del entorno. Un indicio del comportamiento seguro puede resultar de saber lo que una persona hace cuando nadie la ve dado que su comportamiento dependerá, en ese momento, mayormente de las convicciones y valores propios, que del juicio que de sus actuaciones puedan tener los demás.

Otra acción importante en el logro del objetivo del cumplimiento de las guías, normas y procedimientos lo representa la familiarización del cerebro con el comportamiento deseado[12]. La repetición mediante la simulación de situaciones permite que el cerebro reconozca el comportamiento deseado como algo normal o al menos habitual y que el comportamiento contrario a la norma sea reconocido como extraño o fuera de lo normal. Las acciones repetidas generarán el hábito y compartir los hábitos con todo el equipo de trabajo construirá la cultura (Tabla 1).

Igualmente, acciones como firma de compromisos, uso de carteleras, mensajes visuales, señales que recuerden la necesidad del cumplimiento de la norma y la sensación de vigilancia, pueden incentivar la afectiva aplicación de la misma, así como involucrar a los equipos en la construcción de las mismas generará un vínculo afectivo con ésta que propenderá no solo por su cumplimiento, sino por la vigilancia del cumplimiento de los demás.

El uso de incentivos que motiven el cumplimiento de la norma o castiguen el incumplimiento puede inducir comportamientos que generan más registros diligenciados que acciones seguras efectuadas y desmotivar el reporte de eventos adversos[13]. El desarrollo de actividades que ocupan la mente diferente a lo laboral, así como la fatiga del final del día o las jornadas extensas, ofrece al cerebro un esfuerzo adicional al momento de tomar una decisión frente a un evento deshonesto, como la decisión voluntaria y consciente de incumplir una norma.

En resumen, el evento adverso en salud sigue siendo una causa importante de complicaciones en nuestros pacientes. La adopción de estrategias provenientes de otras profesiones u ocupaciones, como aquellas que han logrado mejorar la seguridad en la aviación, para buscar impactar positivamente el comportamiento del personal que trabaja en la atención en instituciones de la salud, por si solas podría no tener el impacto esperado. El incumplimiento voluntario de las guías y normas tiene una relación con la honestidad, por esto es importante establecer el perfil personal deseado al momento de los procesos de selección tanto para el acceso a la formación profesional u ocupacional como para el acceso a plazas laborales con el fin de identificar aquellas personas que jamás seguirán una norma, aquellas que siempre

Tabla 1. Resumen de estrategias para mejorar la adherencia del personal a las guías. Construcción del autor

- Trabajar en el diligenciamiento completo de la historia clínica

- Incorporar las guías como políticas institucionales

- Considerar el perfil de los colaboradores desde el proceso de selección (para estudio y/o trabajo)

- Moderar los incentivos tanto positivos como negativos para el cumplimiento

- Usar estrategias como la simulación para adoctrinamiento y repetición de actividades clave

- Usar medios y estrategias de vigilancia sobre el cumplimiento de las guías (cámaras, carteles recordando y motivando) 
la seguirán y sobre todo, aquellas que lo harán según las circunstancias. Las acciones institucionales para el cumplimiento de las normas y guías deben tener el objetivo de evitar el primer evento. Una vez se presentan el primero y el segundo, es más probable que se presenten nuevas situaciones no deseadas. Las medidas como uso de herramientas visuales en los docu- mentos, pantallas de computador, carteleras y formatos institucionales ayudan a recordar la importancia con el cumplimiento y adherencia. Las actividades repetitivas, aquellas de simulación, las capacitaciones permanentes, sensibilizan el cerebro para reconocer como habitual en lugar de extraño el seguimiento de las guías y normas institucionales.

\section{Referencias}

1. AERONAUTICA CIVIL. [En línea] 27 de 04 de 2018. [Citado el: 14 de 10 de 2018.] http://www. aerocivil.gov.co/prensa/noticias/ Pages/Aeron\%C3\%A1utica-Civil-presenta-informe-final--sobreaccidente-a\%C3\%A9reo-de-laempresa-LaMia-.aspx .

2. J. Bryan Sexton, Patrick Grommes, Enikö Zala-Mezö, Gudela Grote, Robert L. Helmreich and Ruth Häusler. Leadership and co-ordination. [aut. libro] Rainer Dietrich. Group Interaction in High Risk Environments. Oxford: Routledge, 2016.

3. Adverse events recording in electronic health record systems in primary care. Sabine E.M. deHoon, Krin Hek, Liceth Van Dijk, Robert A. Verjeij. 163, 2017, BMC Medical Informatics and Decision Making, Vol. 17.

4. Imhof, Michael, Blondel, Constantijn. Malpractice in Surgery: Safety Culture and Quality Management in the Hospital. Primera. Berlyn: EBSCO Hist, 2013.

5. Suzane Gordon, Patrick Mend- enhall, Bonnie Blair O'connor. What Else Health Care Can Learn From Aviation Teamwork and Safety. Primera. s.I.: EBSCOHost, 2013.

6. Perioperative anesthetic documentation: Adherence to current Australian guidelines. Islam Elhalawani, Simon Jenkins, Nicole Newman. 2, s.l.: Journal of Anesthesiolgy Clinical Pharmacology, 2013, Vol. 29. 211-215.

7. Why don't physicians adhere to guideline recommendations in practice? An analysis of barriers among Dutch general practitioner. Marjolein Lugtenberg, Judith Zegers-van Schaik, Gert P Westert, Jaco S Burguers. 54, 2009, Implementation Science, Vol. 4.

8. Canadian Primary Care Physicians' Attitudes Toward Understanding Clinical Practice Guidelines for Diabetes Screening. Paul E. Alexander, Shelly Anee Li, Marcelo Tonelly, Gorgon Guyat. 6, 2016, Canadian Journal Of Diabetes, Vol. 40, págs. 580585.

9. OMS. Organización Mundial de la Salud. [En línea] 2018. [Citado el: 14 de septiembre de 2018.] http://www.who.int/features/ factfiles/patient_safety/es/.

10. Nidhi Maheshwari, Vineeth Kuman. Military Psychology: Concepts, Trends and Interventions. primera. New Dehli, India : EBSCOhost, 2016.

11. Intrinsic honesty and the prevalence of rule violations across societies. Simon Gachter, Jonathan F. Schultz. 2016, Nature, Vol. 531, págs. 496-499.

12. Kahneman, Daniel. Pensar Rápido, Pensar Despacio. Primera. Bogotá : DEBATE, 2012.

13. Ariely, Dan. Por qué Mentimos en Especial a Nosotros Mismos. Primera. s.I.: Ariel, 2012.

14. Why Don't Physicians Follow Clinical Practice Guidelines? A Framework for Improvement. Michael D. Cabana, Cinthia R. Rand, Neil R. Powe, ALbert W. Wu, Modena H. Wilson, Paul André, Haya R. Rubin. 15, s.l.: JAMA, 1999, Vol. 282.

15. Dietrich, Rainer, GIHRE Project, Childress, Traci Michelle. Group Interaction in High Risk Environments. Oxford: Routledge, 2016, págs. 169-172. 